\title{
Gene segregation in the two-spored basidiomycete Coprinus bilanatus
}

\author{
R. F. O. Kemp
} Department of Botany, University of Edinburgh.
Mayfield Road, Edinburgh EH9 3JH.

\begin{abstract}
The isolation of spore diads of Coprinus bilanatus shows that the proportion of dikaryotic spores can be much higher than the two-thirds expected if the four meiotic products are associated into the two spores formed on each basidium at random. The analysis of spore diads makes it possible to identify some of the spores which are formed with a single nucleus rather than with two nuclei having the same genotype.
\end{abstract}

\section{INTRODUCTION}

In the ascomycetes there are several species which form 4-spored asci instead of asci with eight spores. Neurospora tetrasperma is 4-spored and N. crassa is 8 -spored. In many genera of basidiomycetes there are species which form only two spores on each basidium instead of four. The cultivated mushroom Agaricus bisporus is probably the best known 2-spored basidiomycete but in the genus Coprinus about 10 per cent of the species, out of a total of about 120 species, are 2-spored. One species C. trisporus has 3-spored basidia. All of the species which form 2- or 3-spored basidia are heterothallic and have homogenic mating-type alleles which promote outbreeding. The formation of 2-spored basidia would not be expected in homothallic species as it is a means of producing a high proportion of dikaryotic spores. In this paper the terms homothallic and heterothallic will be applied to species and not to spores. A homothallic species does not have any homogenic self-incompatibility loci whereas a heterothallic species has one or more self-incompatibility loci which promote outbreeding.

One of the earliest studies on a 2-spored basidiomycete was that of Sass (1929) who interpreted the incompatibility system of Coprinus sassii as being unifactorial on the basis of a rather small number of homokaryotic isolates. This species has since been found to have bifactorial incompatibility with clamp connexions (Kemp, 1974). Sass noted that the spindles of the second meiotic division were obliquely placed in the basidium and suggested that the formation of dikaryotic spores might be due to the changed orientation of the second meiotic spindles. Evans (1953) working with the cultivated mushroom noted that the second meiotic spindles were often crossed and orientated in line with the long axis of the basidium. Langton and Elliott (1980) suggested that the dikaryotic spores were formed as a result of the random association of two of the four meiotic products into each spore. They noted that the random migration of the nuclei into the two spores would result in $2 / 3$ rds of the spores being dikaryotic. Elliott and Challen (1983) using Coprinus bilanatus nom. prov., the species also used in this study, found that the proportion of dikaryotic spores, formed by three dikaryons, each heterozygous for a single auxotrophic marker, lay between 51 per cent and 65 per cent. In these experiments random spores were spread on agar and all the colonies were isolated after germination to check for the presence of mycelia with clamp connexions and for the segregation of auxotrophic markers.

In order to distinguish between the random and non-random association of the four meiotic nuclei into the two spores it is necessary to do more than score the relative proportions of dikaryotic to homokaryotic colonies arising from the plating of random spores. The mycelium formed by the germination of a binucleate spore which has two nuclei of the same mating-type factors will be indistinguishable from that formed by a uninucleate spore. If two-thirds of the spores were binucleate and gave rise to dikaryotic mycelia and one-third were uninucleate and formed clampless homokaryotic mycelia this might seem to give strong support to the random migration hypothesis. It would in fact mean that all the spores 
which received two nuclei contained two compatible nuclei; and those which developed into clampless mycelia contained only one nucleus and not two nuclei having the same mating-type alleles. It is therefore necessary to design an experiment which is able to distinguish between homokaryotic spores, which receive two identical nuclei for the markers concerned, and monokaryotic spores which receive only one of the meiotic products.

By isolating spore diads it is possible to distinguish between homokaryotic and monokaryotic spores in diads of two classes. Firstly, a spore diad which germinates to give one clamp-bearing dikaryotic mycelium and one clampless homokaryotic mycelium must have had one uninucleate spore. Secondly when diads are isolated from a dikaryon which is heterozygous for a recessive marker then the appearance of two clampless mycelia in a spore diad, both of which are auxotrophic, must mean that both spores were uninucleate when formed. It is however possible for diads to form one clamp-bearing mycelium and one clampless mycelium if three nuclei migrate into one spore and one into the other. But this still means that the clampless mycelium was formed from a monokaryotic spore and not a homokaryotic spore. All diads which form two clamp-bearing mycelia must have been formed with two compatible nuclei in each spore. As gene segregation and the migration of varying numbers of meiotic nuclei into the two basidiospores formed by certain species of basidiomycete are not frequent topics of discussion the events will be discussed in detail.

Although the basidia form only two spores meiosis is normal in that it forms four haploid meiotic products. If a single pair of alleles is segregating, or if the segregation of several pairs of alleles results in a di-type tetrad being formed, then there will be two nuclei of each genotype. If the diploid nuclei which undergo meiosis are heterozygous for a single pair of alleles $A / a$ then the four nuclei can be numbered and assigned a genotype as follows:

$$
1=A, \quad 2=A, \quad 3=a, \quad 4=a
$$

so that there are three different ways in which these can be combined together in two pairs:

$$
\begin{array}{lll} 
& \text { genotype } & \text { phenotype } \\
1+2: 3+4 & A A+a a & H+H \\
1+3: 2+4 & A a+A a & D+D \\
1+4: 2+3 & A a+A a & D+D
\end{array}
$$

If one nucleus fails to migrate from the basidium then the following types of spore diads will be formed if nucleus number 4 is left behind:

$$
\begin{array}{lll}
1+2: 3 & A A+a & H+M \\
1+3: 2 & A a+A & D+M \\
2+3: 1 & A a+A & D+M
\end{array}
$$

The same pattern will result if the other nuclei are left behind in turn. If two meiotic products fail to migrate then two monokaryotic spores will be formed. The expected results for random and nonrandom migration of differing numbers of nuclei are shown in fig. 1.

Fig. I(a) shows the relative proportions of clamp-bearing $\left(c^{\dagger}\right)$ to clampless $(c)$ mycelia expected by the random migration of four or three or two nuclei into the two spores. Fig. 1(b) shows (a)

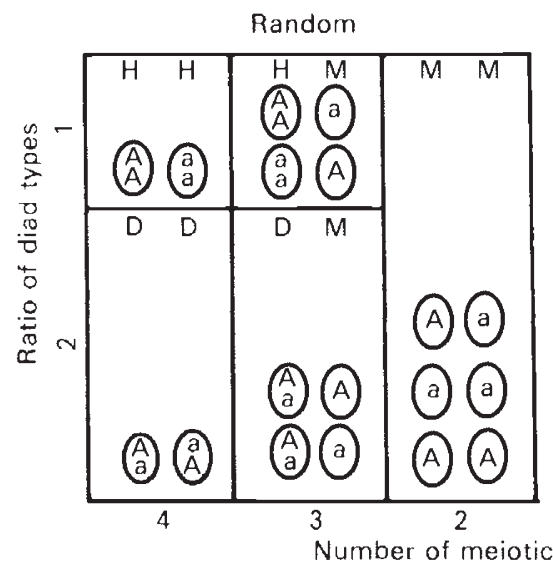

(b)

Non random

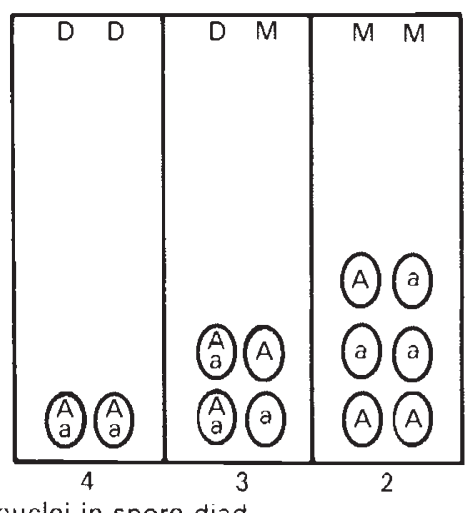

Figure 1 The consequences of random (a) and non-random (b) migration of four, three or two meiotic nuclei into basidiospore diads for a single factor $A / a$. 
the effect of non-random migration of differing numbers of nuclei into the two spores. The random migration of two nuclei into each of the two spores will result in two-thirds of the spore diads having two dikaryotic spores, both of which will develop into clamp-bearing dikaryotic mycelia. The random migration of three nuclei into the two spores would result in two-thirds of the diads having one dikaryotic and one monokaryotic spore and the remaining one-third would form two clampless mycelia. These diads will consist of one spore which has two nuclei with the same incompatibility alleles and another spore with only a single nucleus. If only two nuclei migrate from the basidium into the two spores then both will be monokaryotic and form clampless homokaryotic mycelia.

In contrast the non-random migration of the various numbers of nuclei could result in all of the spore diads, which receive a given number of nuclei, being the same (fig. 1(b)). It is essential to distinguish between dikaryotic spores which receive two compatible nuclei; homokaryotic spores which have two nuclei with the same mating type alleles and monokaryotic spores which contain only one nucleus when formed. The nonrandom association of the four haploid meiotic nuclei into two spores could give 100 per cent dikaryotic spores.

\section{MATERIALS AND METHODS}

Coprinus bilantus nom. prov. was chosen for the study of gene segregation in a 2-spored basidiomycete because its mycelia grow well and fruit in culture and it has bifactorial incompatibility with clamp-connexions (Kemp, 1974). This is the system of homogenic incompatibility found in $C$. cinereus and in Schizophyllum commune. It has good spore germination and it is relatively easy to distinguish between dikaryons, common-B heterokaryons and homokaryons. In this paper the term monokaryotic spore will be used to refer to a spore which receives only one of the haploid meiotic products. The term homokaryotic spore will refer to a spore which receives two nuclei both of which have the same mating-type alleles. A homokaryotic mycelium contains nuclei of only one genotype in contrast to a dikaryotic mycelium which contains nuclei of two compatible genotypes. A heterokaryotic mycelium contains nuclei of two genotypes which are not fully compatible; for example common-B heterokaryons $(A 1 B 1 / A 2 B 1)$ and common-A heterokaryons $(A 1 B 1 / A 1 B 2)$. The common-B heterokaryons can be distinguished by their formation of a streaky mycelium with false clamp connexions at many septa. The common-A heterokaryons can only be identified with certainty by doing mating tests.

The first isolates of the new species $C$. bilanatus were obtained from Holland where they were found growing in cucumber houses by Dr J. Daams of Kortenhoef (Kemp, 1974). Since then isolates have been received from Angola, Java, Calcutta and Israel but not from the British Isles. All of the isolates interbreed with each other but not with any other species of Coprinus in the section Lanatuli (Kemp, 1975). C. bilanatus is the only 2 -spored species in this section of the genus apart from a single isolate obtained from Japan. The Japanese isolate has larger and more pointed spores and will not interbreed with any isolates of C. bilanatus. The isolates are listed in table 1 .

All cultures were grown on horse-dung extract agar (Lange, 1952). Most isolates fruited well on this medium but some did better on a mixture of sterile straw and dung prepared in milk bottles. Spore diads were isolated with a micro-needle, rather than a loop, using a Saunders-Singer

Table 1 The origin of the isolates of $C$. bilanatus and their genotypes

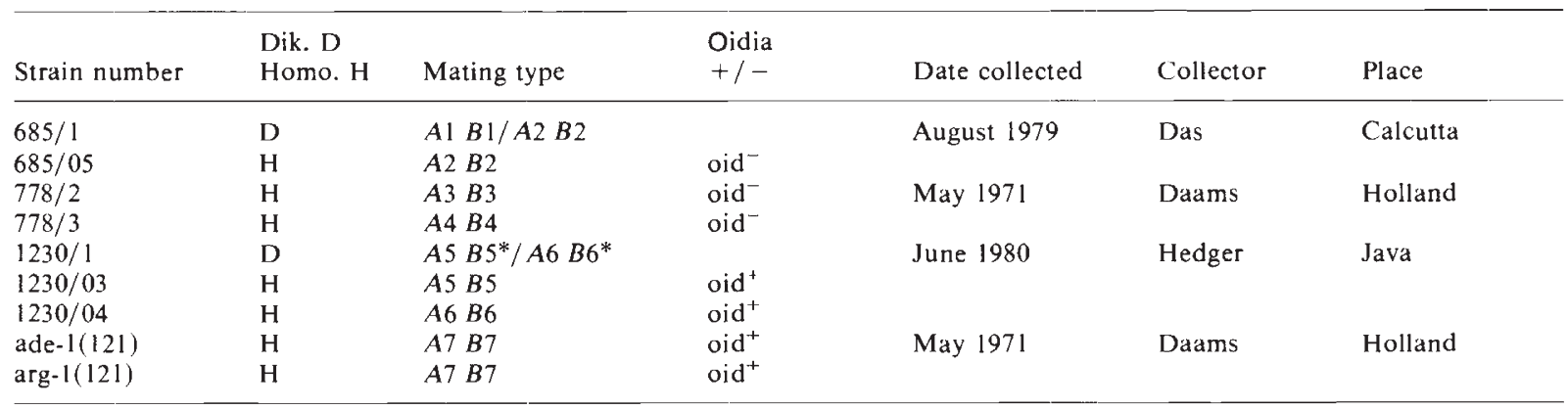

* Cycloheximide resistant. 
micromanipulator. The medium used for the germination of spores was prepared without horsedung extract as its inclusion was found to inhibit spore germination. Mycelial cultures were incubated at $25^{\circ} \mathrm{C}$ and those for fruiting were kept at room temprature $\left(20-23^{\circ} \mathrm{C}\right)$ in diffuse light.

The spore diads were isolated onto small blocks of agar $6 \mathrm{~mm} \times 3 \mathrm{~mm} \times 3 \mathrm{~mm}$ thick. After incubation for 1 day the blocks were examined for spore germination and divided into two and placed some distance apart on fresh plates or transferred onto slopes. After 4-5 days incubation the colonies were examined for clamp-connexions. The segregation of auxotrophic markers was tested on the minimal medium of Raper et al. (1972). The isolate obtained from Java was cycloheximide resistant and this was added to the media at a concentration of $10 \mu \mathrm{g} \mathrm{ml}^{-1}$.

Homokaryotic mycelia were obtained from dikaryons by maceration using a test-tube grinder sterilised in alcohol and dried over a bunsen flame. A $2-3 \mathrm{~mm}$ cube of agar and hyphae was placed in $4-5 \mathrm{ml}$ of sterile water in the tube and the plunger pressed down 6-10 times. The amount of maceration needed varies with the fit of the plunger in each tube. The plunger was not rotated as it is essential not to homogenise the hyphae into short inviable fragments. The suspension of hyphal fragments was transferred to plates of medium on the end of the plunger. The transfer of one drop onto one plate and two drops onto another was usually adequate. After 2-3 days incubation inocula from about 20 of the smallest colonies were transferred onto fresh plates and incubated for a further 3-4 days. They were then checked for the presence or absence of clamp connexions. By this method it is possible to do tetrad analysis despite the fact that each basidium forms only two spores.

\section{RESULTS}

The results for spore diads isolated from wild dikaryotic strains are shown in table 2(a) and are listed as experiments 1-4. Those for dikaryons using the auxotrophic markers produced by Elliott are shown in table 2(b), experiments 5-9. A total of 459 diads was isolated and of these only 18 failed to germinate to give two mycelia. The incomplete diads are not included in the totals shown in table 2.

\section{DISCUSSION}

Table 2(a) clearly shows that the number of homokaryotic spores formed is considerably less than the one-third expected if the two nuclei in each spore become associated at random. Only $5 \cdot 2$ per cent of the diads could possibly have contained two binucleate spores which were homozygous for the mating-type alleles. These diads could also have been formed with two uninucleate spores. If the spores in these diads had been plated at random then about 90 per cent would have developed into dikaryotic mycelia. None of the diads germinated to give two mycelia which had the streaky growth form of common- $B$ heterokaryons

Table 2 The numbers of spore diads consisting of two dikaryotic spores (I)I)); one dikaryotic spore and one monokaryotic spore (DM) and two monokaryotic spores (MM) in dikaryons isolated from the wild (a) and from dikaryons heterozygous for an auxotrophic marker (b)

\begin{tabular}{|c|c|c|c|c|c|c|}
\hline Expt. & Strain & $\begin{array}{l}\text { Total } \\
\text { complete diads }\end{array}$ & $\mathrm{DD}(\%)$ & $\operatorname{DM}(\%)$ & $\mathrm{MM}(\%)$ & $\begin{array}{l}\text { Incomplete } \\
\text { diads }\end{array}$ \\
\hline \multicolumn{7}{|l|}{ (a) } \\
\hline 1 & $1230 / 1$ & 100 & $85(85)$ & $9(9)$ & $6(6)$ & 2 \\
\hline 2 & $685 / 1$ & 19 & $18(94 \cdot 7)$ & $1(5 \cdot 3)$ & $0(0)$ & 1 \\
\hline 3 & $1230 / 04 \times 778 / 4$ & 19 & $17(89 \cdot 5)$ & () $(0)$ & $2(10 \cdot 5)$ & 0 \\
\hline \multirow[t]{2}{*}{4} & $1230 / 04 \times 778 / 2$ & 71 & $58(81 \cdot 7)$ & $10(14)$ & $3(4 \cdot 3)$ & 4 \\
\hline & Totals & 209 & $178(85 \cdot 2)$ & $20(9 \cdot 6)$ & $11(5 \cdot 2)$ & 7 \\
\hline \multicolumn{7}{|l|}{ (b) } \\
\hline 5 & $685 / 05 \times$ ade -1 & 35 & $32(91 \cdot 4)$ & $2(5 \cdot 8)$ & $1(2 \cdot 8)$ & () \\
\hline 6 & $1230 / 04 \times$ ade -1 & 56 & $36(64 \cdot 3)$ & $8(14: 3)$ & $12(21 \cdot 4)$ & 0 \\
\hline 7 & $1230 / 03 \times$ adc- 1 & 14 & $8(57)$ & $5(36)$ & $1(7)$ & 3 \\
\hline 8 & $1230 / 03 \times \arg$ & 56 & $28(50)$ & $10(18)$ & $18(32)$ & 8 \\
\hline \multirow[t]{2}{*}{9} & $778 / 2 \times$ arg & 71 & $34(48)$ & $15(21)$ & $22(31)$ & 0 \\
\hline & Totals & 232 & $138(59 \cdot 5)$ & $40(17 \cdot 2)$ & $54(23 \cdot 3)$ & 11 \\
\hline
\end{tabular}


( $A 1 B 1 / A 2 B 1$ ). This suggests that both of the incompatibility loci always segregated at the first meiotic division to give ditype tetrads. In all of the $11 M M$ diads (table 2(a)) the two homokaryotic mycelia formed dikaryotic mycelia when mated together. This could be interpreted as showing that the diad consisted of two uninucleate spores which had complementary genotypes. This might indicate that the first nuclei to migrate into the spores were of opposite mating types (e.g., $A 1 B 1$ and $A 2 B 2$ ). They could also have been formed with two identical nuclei in each spore (e.g., $A 1 B 1+A 1 B 1$ and $A 2 B 2+A 2 B 2$ ).

In experiment four the strain $1230 / 04$ was cycloheximide resistant and $778 / 2$ was cycloheximide sensitive so the dikaryon was heterozygous for a pair of alleles other than those for mating-type. Out of a total of $58 \mathrm{DD}$ diads 10 had one mycelium which was cycloheximide resistant and the other cycloheximide sensitive. In addition in four out of $10 \mathrm{DM}$ diads the dikaryotic mycelium was homozygous for a cycloheximide allele. Out of a total of 68 diads 14 were therefore homozygous at the cycloheximide locus which indicates that in the formation of $28 / 68$ diads the cycloheximide alleles segregated at the second meiotic division.

The results for dikaryons consisting of one wild-type monokaryon and one containing a mutant allele produced by Elliott are shown in table 2(b). Although experiment five shows over 90 per cent of the diads to be DD experiments 6-9 have much lower proportions of DD diads than those shown in table 2(a). The reasons for this are not clear but it explains why Elliott and Challen (1983) got results which differ from those shown in table 2(a). The summed results for experiments 5-9 show that $23 \cdot 3$ per cent of the diads could have contained two homokaryotic spores. If the spores had been plated at random then $68 \cdot 1$ per cent $\left(59 \cdot 5+\frac{1}{2}(17 \cdot 2)\right)$ would have developed into homokaryotic mycelia. In experiment nine the formation of $22 \mathrm{MM}$ diads out of a total of 71 isolated provided a sufficient number to see if any $\mathrm{MM}$ diads developed into two auxotrophic mycelia. Out of $22 \mathrm{MM}$ diads three had two auxotrophic spores and therefore must have been uninucleate when formed. Clearly two of the meiotic products may fail to migrate into the spores during diad formation.

The results for dikaryons isolated from the wild show that the migration of the four nuclei into the two spores does not occur at random. All of the spores germinating to give clampless homokaryotic mycelia could have been uninucleate when formed. The biometrical analysis of Kirby (1983) based on the random association of the four meiotic products into the two spores of the diads is probably therefore not applicable to natural populations of 2 -spored basidiomycetes. Although it is possible to prove that the two spores of a diad were uninucleate in certain classes of diad, it is not possible to prove that a spore diad which germinates to give two homokaryotic mycelia consists of two binucleate spores when formed. By plating random spores it is not possible to distinguish between monokaryotic and homokaryotic spores and this results in an over estimation of the number of homokaryotic spores. The segregation of the $A$ and the $B$ homogenic mating type alleles never showed tetratype segregation and the parental ditypes and non-parental ditypes were formed in roughly equal numbers. This indicates that the two loci are unlinked but that both are closely linked to their respective centromeres. Although the mechanism which determines the high proportion of dikaryotic spores is not known it is clearly not dependent on the random segregation of alleles or the random migration of the nuclei into the spores.

Acknowledgements I thank T. J. Elliott for the auxotrophic strains and also Dr J. Daams, Kortenhoef, Holland who first sent me this useful new species. Thanks are also due to the other collectors and the SERC for a grant. The referee is thanked for his remodelling of the text leading up to fig. 1.

\section{REFERENCES}

ELliotr, T. J. AND ChALlEN, M. P. 1983. Genetic ratios in secondary homothallic basidiomycetes. Exptl. Mycol., 7, $170-174$

EVANS, H. J. 1959. Nuclear behaviour in the cultivated mushroom. Chromosoma, 10, 115-135.

KEMP, R. F. O. 1974. Bifactorial incompatibility in the twospored basidiomycetes Coprinus sassii and $C$. bilantus. Trans. Brit. mycol. Soc., 62, 547-555.

KEMP, R. F. O. 1975. Breeding biology of Coprinus species in the section Lanatuli. Trans. Brit. mycol. Soc., 65, 375-388.

KIRBY, G. C. 1983. Breeding systems and heterozygosity in populations of tetrad forming fungi. Heredity, 52, 35-41.

LANGE, M. 1952. Species concept in the genus Coprinus, a study of the significance of intersterility. Dansk. bot. Ark., 14, $1-164$.

LANGTON, F. A. AND ELLIOTT, T. J. 1980. Genetics of secondary homothallic basidiomycetes. Heredity, 45, 99-106.

RAPER, (. A., RAPER, J. R. ANI) MILLER, R. E. 1972. Genetic analysis of the life-cycle of Agaricus bisporus. Mycologia, $64,1088-1177$.

SASS, J. E. 1929. The cytological basis for homothallism and heterothallism in the Agaricaceae. Am. J. Bot., 16, 663-701. 